\title{
Summary DOE Spent Fuel Report
}

\author{
Layne Pincock
}

April 2017

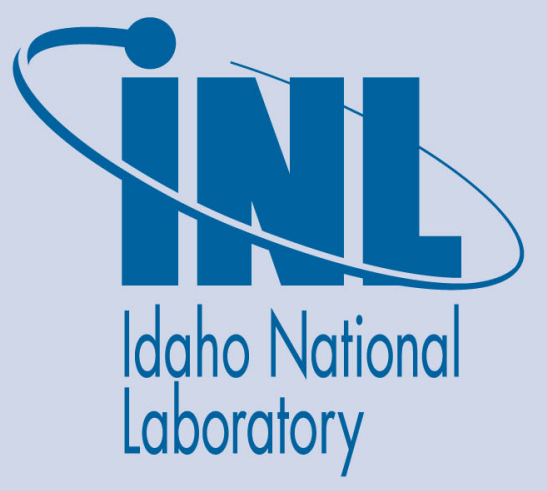

The INL is a U.S. Department of Energy National Laboratory operated by Battelle Energy Alliance 
INL/EXT-17-41890-Revision-0

\title{
Summary DOE Spent Fuel Report
}

\author{
Layne Pincock
}

April 2017

Idaho National Laboratory Idaho Falls, Idaho 83415

http://www.inl.gov

Prepared for the U.S. Department of Energy Office of Environmental Management Under DOE Idaho Operations Office Contract DE-AC07-05ID14517 
Spent Fuel Management Facilities Government ${ }^{1}$ Facilities

\begin{tabular}{|c|c|c|c|c|c|c|c|c|c|}
\hline State & Installation & Facility & Function & Licensee & Regulator & SF Source ${ }^{2}$ & Inventory & Units & $\begin{array}{l}\text { Estimated } \\
\text { Activity }(\mathrm{Bq}) \text { for } \\
2017^{4}\end{array}$ \\
\hline \multirow[t]{2}{*}{ Colorado } & $\begin{array}{l}\text { U.S. Geological Survey } \\
\text { (Denver) }\end{array}$ & $\begin{array}{l}\text { Research/Test } \\
\text { Reactor }\end{array}$ & Wet Storage & U.S. Geological Survey & NRC & 2 & 0.04 & MTHM & $5.35 E+14$ \\
\hline & Fort St. Vrain & ISFSI & Dry Storage & $\mathrm{DOE}$ & NRC & 2 & 14.73 & MTHM & $1.53 \mathrm{E}+17$ \\
\hline \multirow[t]{3}{*}{ Idaho } & \multirow[t]{3}{*}{ Idaho National Lab } & \begin{tabular}{|l} 
Multiple INL \\
Facilities \\
\end{tabular} & Wet Storage & DOE & DOE & 1,2 & 2.93 & MTHM & $1.58 \mathrm{E}+17$ \\
\hline & & \begin{tabular}{|l} 
Multiple INL \\
Facilities \\
\end{tabular} & Dry Storage & DOE & DOE & 1,2 & 195.74 & MTHM & $1.70 \mathrm{E}+18$ \\
\hline & & ISFSI $^{3}$ & Dry Storage & DOE & NRC & 2 & 81.59 & MTHM & $9.25 E+16$ \\
\hline Illinois & Argonne National Lab & $\begin{array}{l}\text { ANL Spent Fuel } \\
\text { Storage }\end{array}$ & Dry Storage & DOE & DOE & 1,2 & 0.12 & MTHM & $3.90 E+15$ \\
\hline \multirow[t]{2}{*}{ Maryland } & $\begin{array}{l}\text { National Institute of } \\
\text { Standards and Technology } \\
\text { (Gaithersburg) }\end{array}$ & $\begin{array}{l}\text { Research/Test } \\
\text { Reactor }\end{array}$ & Wet Storage & $\begin{array}{l}\text { National Institute of } \\
\text { Standards and } \\
\text { Technology } \\
\end{array}$ & NRC & 2 & 0.02 & MTHM & $1.14 \mathrm{E}+16$ \\
\hline & $\begin{array}{l}\text { Armed Forces Radiobiology } \\
\text { Research Institute (Bethesda) }\end{array}$ & $\begin{array}{l}\text { Research/Test } \\
\text { Reactor }\end{array}$ & Wet Storage & $\begin{array}{l}\text { Armed Forces } \\
\text { Radiobiology Research } \\
\text { Institute }\end{array}$ & NRC & 1 & 0.02 & MTHM & $3.03 E+14$ \\
\hline New Mexico & Sandia National Lab & \begin{tabular}{|l} 
Multiple SNL \\
Facilities \\
\end{tabular} & Dry Storage & DOE & DOE & 1,2 & 0.24 & MTHM & $5.89 E+16$ \\
\hline Rhode Island & $\begin{array}{l}\text { Rhode Island Atomic Energy } \\
\text { Commission (Narragansett) }\end{array}$ & $\begin{array}{l}\text { Research/Test } \\
\text { Reactor }\end{array}$ & Wet Storage & $\begin{array}{l}\text { Rhode Island Atomic } \\
\text { Energy Commission }\end{array}$ & NRC & 2 & 0.02 & MTHM & $1.63 E+14$ \\
\hline \multirow[t]{2}{*}{$\begin{array}{l}\text { South } \\
\text { Carolina }\end{array}$} & \multirow[t]{2}{*}{ Savannah River Site } & \multirow[t]{2}{*}{ SRS Storage } & Wet Storage & DOE & DOE & 1,2 & 29.42 & MTHM & $1.78 \mathrm{E}+18$ \\
\hline & & & Dry Storage & DOE & DOE & 2 & 0.01 & MTHM & $2.96 \mathrm{E}+15$ \\
\hline Tennessee & Oak Ridge Reservation & $\begin{array}{l}\text { Multiple ORR } \\
\text { Facilities }\end{array}$ & Wet Storage & DOE & DOE & 2 & 0.47 & MTHM & $2.86 \mathrm{E}+17$ \\
\hline \multirow[t]{2}{*}{ Washington } & \multirow[t]{2}{*}{ Hanford Site } & \multirow[t]{2}{*}{$\begin{array}{l}\text { Multiple Hanford } \\
\text { Facilities }\end{array}$} & Dry Storage & DOE & DOE & 1,2 & 2127.88 & MTHM & $1.79 \mathrm{E}+18$ \\
\hline & & & Wet Storage & DOE & DOE & 1 & 1.50 & MTHM & $9.19 E+14$ \\
\hline
\end{tabular}

${ }^{1}$ Data Source: DOE National Spent Nuclear Program database (SFD Version 6.2.3, 3/24/2011) except where noted. Navy Spent Fuel is excluded from this report.

${ }^{2}$ SF Sources: 1-Defense applications; 2-Commercial NPPs and Test/Research Reactors.

${ }^{3}$ In addition to this facility, NRC licensed a second DOE ISFSI at Idaho, which DOE subsequently decided not to construct.

${ }^{4}$ Source Term estimates calculated for 2017 based on methodology described in report DOE/SNF/REP-078 Rev. 2 "Source Term Estimates for DOE SNF". 
Spent Fuel Management Facilities University Research Facilities ${ }^{5}$

\begin{tabular}{|c|c|c|c|c|c|c|c|c|}
\hline State & Installation & Facility & Function & Licensee & Regulator & Inventory & Units & $\begin{array}{l}\text { Estimated } \\
\text { Activity (Bq) for } \\
2017^{7}\end{array}$ \\
\hline \multirow[t]{2}{*}{ California } & University of California (Irvine) & Research Reactor & Wet Storage & University of California & NRC & 21.42 & $\mathrm{kgU}$ & $9.85 \mathrm{E}+13$ \\
\hline & University of California (Davis) $^{6}$ & Research Reactor & Wet Storage & University of California & NRC & 72.31 & $\mathrm{kgU}$ & $9.92 \mathrm{E}+14$ \\
\hline Florida & University of Florida (Gainesville) & Research Reactor & Wet Storage & University of Florida & NRC & 23.81 & $\mathrm{kgU}$ & $1.38 \mathrm{E}+14$ \\
\hline Indiana & Purdue University (West Lafayette) & Research Reactor & Wet Storage & Purdue University & NRC & 17.53 & $\mathrm{kgU}$ & $2.12 \mathrm{E}+14$ \\
\hline Kansas & Kansas State University (Manhattan) & Research Reactor & Wet Storage & Kansas State University & NRC & 21.08 & $\mathrm{kgU}$ & $5.23 E+14$ \\
\hline Maryland & University of Maryland (College Park) & Research Reactor & Wet Storage & University of Maryland & NRC & 16.35 & $\mathrm{kgU}$ & $4.95 \mathrm{E}+14$ \\
\hline \multirow[t]{3}{*}{ Massachusetts } & University of Lowell (Lowell) & Research Reactor & Wet Storage & University of Lowell & NRC & 10.13 & $\mathrm{kgU}$ & $1.23 \mathrm{E}+14$ \\
\hline & \begin{tabular}{|l|}
$\begin{array}{l}\text { Massachusetts Institute of Technology } \\
\text { (Cambridge) }\end{array}$ \\
\end{tabular} & Research Reactor & Wet Storage & $\begin{array}{l}\text { Massachusetts Institute } \\
\text { of Technology }\end{array}$ & NRC & 19.85 & $\mathrm{kgU}$ & $5.77 \mathrm{E}+15$ \\
\hline & $\begin{array}{l}\text { Worcester Polytechnic Institute } \\
\text { (Worcester) }\end{array}$ & Research Reactor & Wet Storage & $\begin{array}{l}\text { Worcester Polytechnic } \\
\text { Institute }\end{array}$ & NRC & 22.75 & $\mathrm{kgU}$ & $1.40 \mathrm{E}+13$ \\
\hline \multirow[t]{2}{*}{ Missouri } & University of Missouri (Columbia) & Research Reactor & Wet Storage & University of Missouri & NRC & 35.44 & $\mathrm{kgU}$ & $3.18 \mathrm{E}+15$ \\
\hline & University of Missouri (Rolla) & Research Reactor & Wet Storage & University of Missouri & NRC & 26.46 & $\mathrm{kgU}$ & $3.29 \mathrm{E}+15$ \\
\hline North Carolina & North Carolina State University (Raleigh) & Research Reactor & Wet Storage & $\begin{array}{l}\text { North Carolina State } \\
\text { University }\end{array}$ & NRC & 315.40 & $\mathrm{kgU}$ & $6.22 \mathrm{E}+14$ \\
\hline Ohio & Ohio State University (Columbus) & Research Reactor & Wet Storage & Ohio State University & NRC & 26.15 & $\mathrm{kgU}$ & $3.16 \mathrm{E}+14$ \\
\hline \multirow[t]{2}{*}{ Oregon } & Oregon State University (Corvallis) & Research Reactor & Wet Storage & Oregon State University & NRC & 77.06 & $\mathrm{kgU}$ & $8.78 \mathrm{E}+14$ \\
\hline & Reed College (Portland) & Research Reactor & Wet Storage & Reed College & NRC & 30.86 & $\mathrm{kgU}$ & $4.34 \mathrm{E}+14$ \\
\hline Pennsylvania & $\begin{array}{l}\text { Pennsylvania State University (University } \\
\text { Park) }\end{array}$ & Research Reactor & Wet Storage & $\begin{array}{l}\text { Pennsylvania State } \\
\text { University }\end{array}$ & NRC & 37.57 & $\mathrm{kgU}$ & $1.38 \mathrm{E}+15$ \\
\hline \multirow[t]{2}{*}{ Texas } & Texas A\&M University (College Station) & Research Reactor & Wet Storage & Texas A\&M University & NRC & 70.76 & $\mathrm{kgU}$ & $1.30 \mathrm{E}+15$ \\
\hline & University of Texas (Austin) & Research Reactor & Wet Storage & University of Texas & NRC & 35.17 & $\mathrm{kgU}$ & $2.73 \mathrm{E}+14$ \\
\hline Utah & University of Utah (Salt Lake City) & Research Reactor & Wet Storage & University of Utah & NRC & 26.82 & $\mathrm{kgU}$ & $1.22 \mathrm{E}+15$ \\
\hline Washington & Washington State University (Pullman) & Research Reactor & Wet Storage & $\begin{array}{l}\text { Washington State } \\
\text { University }\end{array}$ & NRC & 67.48 & $\mathrm{kgU}$ & $3.06 \mathrm{E}+15$ \\
\hline Wisconsin & University of Wisconsin (Madison) & Research Reactor & Wet Storage & University of Wisconsin & NRC & 68.06 & $\mathrm{kgU}$ & $4.34 \mathrm{E}+14$ \\
\hline
\end{tabular}

${ }^{5}$ Data Source: DOE National Spent Nuclear Program database (SFD Version 6.2.3, 3/24/2011) except where noted.

${ }^{6}$ Formerly McClellan AFB (Sacramento).

${ }^{7}$ Source Term estimates calculated for 2017 based on methodology described in report DOE/SNF/REP-078 Rev. 2 "Source Term Estimates for DOE SNF". 
Spent Fuel Management Facilities Other Research and Nuclear Cycle Facilities ${ }^{8}$

\begin{tabular}{|c|c|c|c|c|c|c|c|c|}
\hline State & Installation & Facility & Function & Licensee & Regulator & Inventory & Units & \begin{tabular}{|l|} 
Estimated \\
Activity (Bq) for \\
$2017^{9}$
\end{tabular} \\
\hline \multirow[t]{2}{*}{ California } & Aerotest Research (San Ramon) & Research/Test Reactor & Wet Storage & Aerotest Research & NRC & 17.43 & $\mathrm{kgU}$ & $3.54 \mathrm{E}+15$ \\
\hline & General Electric (Pleasanton) & Research/Test Reactor & Wet Storage & General Electric & NRC & 3.86 & $\mathrm{kgU}$ & $5.11 \mathrm{E}+12$ \\
\hline Michigan & Dow Chemical Co. (Midland) & Research/Test Reactor & Wet Storage & Dow Chemical Co. & NRC & 14.81 & $\mathrm{kgU}$ & $2.23 E+14$ \\
\hline Virginia & BWX Technology, Inc. & Fuel Cycle Facility & Dry Storage & BWX Technology & NRC & 43.48 & $\mathrm{kgU}$ & $2.15 \mathrm{E}+15$ \\
\hline
\end{tabular}

${ }^{8}$ Data Source: DOE National Spent Nuclear Program database (SFD Version 6.2.3, 3/24/2011) except where noted.

${ }^{9}$ Source Term estimates calculated for 2017 based on methodology described in report DOE/SNF/REP-078 Rev. 2 "Source Term Estimates for DOE SNF". 


\begin{tabular}{|c|c|c|c|c|c|}
\hline Site & MTHM & $\begin{array}{l}\text { Volume in Cubic } \\
\text { Meters }\end{array}$ & Canisters & $\begin{array}{l}\text { Commercial } \\
\text { Assembly } \\
\text { Equivalent }^{2}\end{array}$ & Curies $^{3}$ \\
\hline Hanford & 2130.4 & 228.4 & 560 & 1248 & $3.83 E+07$ \\
\hline Idaho $^{1}$ & 322.9 & 539.3 & 1704 & 2947 & $5.21 \mathrm{E}+07$ \\
\hline Savannah River & 29.4 & 64 & 614 & 350 & $2.70 \mathrm{E}+07$ \\
\hline Other & 23.8 & 20.3 & 913 & 111 & $3.20 E+07$ \\
\hline TOTAL & 2506.5 & 852 & 3791 & 4656 & $1.49 E+08$ \\
\hline
\end{tabular}

\begin{tabular}{|c|c|c|c|c|c|}
\hline Site & MTHM (\%) & $\begin{array}{l}\text { Volume in Cubic } \\
\text { Meters (\%) }\end{array}$ & Canisters (\%) & $\begin{array}{l}\text { Commercial } \\
\text { Assembly } \\
\text { Equivalent }{ }^{2}(\%)\end{array}$ & Curies $^{3}(\%)$ \\
\hline Hanford & $85 \%$ & $27 \%$ & $15 \%$ & $27 \%$ & $26 \%$ \\
\hline Idaho $^{1}$ & $13 \%$ & $63 \%$ & $45 \%$ & $63 \%$ & $35 \%$ \\
\hline Savannah River & $1 \%$ & $8 \%$ & $16 \%$ & $8 \%$ & $18 \%$ \\
\hline Other & $1 \%$ & $2 \%$ & $24 \%$ & $2 \%$ & $21 \%$ \\
\hline TOTAL & 2506.5 & 852 & 3791 & 4656 & $1.49 \mathrm{E}+08$ \\
\hline
\end{tabular}

${ }^{1}$ Includes FSV, excludes Navy

${ }^{2}$ Volume equivalent to typical (17x17) LWR fuel assembly $\left(4 \mathrm{~m} \times 0.214 \mathrm{~m} \times 0.214 \mathrm{~m}=0.183 \mathrm{~m}^{3}\right)$

${ }^{3} 2030$ Nominal Estimates

Source: SFD v6.2.3 (3-24-2011). 